\title{
Extracellular vesicles from mesenchymal stem cells of dental pulp and adipose tissue display distinct transcriptomic characteristics suggestive of potential therapeutic targets
}

\author{
Terunuma $\mathrm{A}^{1}$, Yoshioka $\mathrm{Y}^{2}$, Sekine $\mathrm{T}^{3}$, Takane $\mathrm{T}^{3}$, Shimizu $\mathrm{Y}^{3}$, Narita $\mathrm{S}^{3}$, Ochiya $\mathrm{T}^{2}$, Terunuma $\mathrm{H}^{3,4}$
}

\begin{abstract}
Objective: Mesenchymal stem cells (MSCs) are isolated from various human tissues and used for therapy, in which beneficial effects are attributed mainly to mesenchymal stem cell-derived extracellular vesicles (MSC-EVs). Whereas MSCs of diverse tissue types share cardinal stem cell features, it is becoming evident that MSCs of each tissue type possess unique properties as well. For designing efficient stem cellbased therapies, it is crucial to understand the unique properties associated with MSCs and MSC-EVs of each tissue type. Such unique properties can be analyzed through transcriptomic approaches using comprehensive gene expression databases and sophisticated analytical tools. Here, we comparatively studied the transcriptomes in MSC-EVs of dental pulp and adipose tissue. Additionally, the transcriptomes of MSC-EVs were compared with the cellular transcriptomes of MSCs for the same tissue types.

Methods: MSCs were cultured from human dental pulp and adipose tissue specimens. Conditioned culture media were collected to prepare MSC-EVs, from which RNAs were isolated and subjected to next-generation sequencing for transcriptomic analysis. Gene expression signatures in MSC-EVs of each tissue type were investigated using gene set analysis.

Results: MSC-EVs obtained from dental pulp-derived MSCs showed distinct transcriptomic signatures of neurogenesis and neural retina development while MSC-EVs of adipose tissue-derived MSCs showed signatures of mitochondrial activity and skeletal system development. The transcriptomes of MSC-EVs resembled the cellular transcriptomes of MSCs, and the genes associated with neurogenesis were highly expressed in both MSCs and MSC-EVs of dental pulp. Adipose tissue-derived MSCs and MSC-EVs highly expressed genes associated with angiogenesis, hair growth, and dermal matrices.

Conclusion: The clear and distinct signatures of neurogenesis and neural retina development in dental pulp-derived MSC-EVs imply neurodegenerative disorders and retinal diseases as putative therapeutic targets. In contrast, the transcripts in adipose tissue-derived MSC-EVs could be useful in rejuvenating the skin and musculoskeletal system. Further insights into MSC-EVs of divergent tissue types may expand the list of potential therapeutic targets.
\end{abstract}

Keywords: Exosome; Transcriptome; Gene set analysis; Neurogenesis; Angiogenesis; Neural retina; Hair growth; Conditioned media

\section{Introduction}

MSCs are isolated from divergent adult tissues for clinical applications to demonstrate promising therapeutic potentials $^{[1,2]}$. Many studies suggest that MSCs exert beneficial effects via secreted factors including extracellular vesicles $(E V s)^{[3,4]}$. Whereas MSCs from various tissues share common stem cell markers and biological behaviors, they also appear to possess unique properties depending on the tissue types they originated from ${ }^{[5,6]}$. Diversity of MSCs from different tissue types may result in various efficacies of MSCs in treating a disease of interest ${ }^{[7,8]}$, and the MSCs with the highest efficacy should be chosen for successful stem cell-based therapies ${ }^{[9]}$. Considering the pivotal role of EVs in exerting therapeutic effects of MSCs, it is crucial to understand the unique properties of MSC-EVs of various tissue types so that MSC-EVs with the most desirable properties are chosen for the therapy. MSCs and MSCEVs can be characterized by analyzing proteins, microRNAs and mRNAs.
Previously, we chose to analyze mRNAs in MSCs by taking advantage of comprehensive transcriptome databases and powerful analytical tools. Transcriptomes in MSCs of dental pulp and adipose tissue displayed unique transcriptomic characteristics for the MSCs of each tissue type ${ }^{[10]}$. In the current study, we investigate whether MSC-EVs of dental pulp and adipose tissue show distinct transcriptomes, and whether MSC-EVs and MSCs of the same tissue type share the transcriptomic characteristics.

\section{Materials and methods \\ Cell sources and extracellular vesicle preparation}

Human adipose tissues and exfoliated deciduous teeth were obtained from healthy donors with written informed consent. MSCs were cultured from the tissues to collect conditioned media at passage 7 . Ninety-six $\mathrm{ml}$ of the MSC conditioned media were centrifuged at $2,000 \times \mathrm{g}$ for 10 minutes and filtered through a

Author Names in full: Atsushi Terunuma ${ }^{1}$, Yusuke Yoshioka ${ }^{2}$, Tatsuya Sekine ${ }^{3}$, Tsubasa Takane ${ }^{3}$, Yoshihisa Shimizu ${ }^{3}$, Satoko Narita $^{3}$, Takahiro Ochiya $^{2}$, Hiroshi Terunuma $^{3,4}$ 
$0.22 \mu \mathrm{m}$ pore-sized membrane (Stericup Quick Release Durapore, Merck Millipore, MA, USA). The filtrates were ultracentrifuged at $210,000 \times \mathrm{g}(35,000 \mathrm{rpm})$ using a SW41Ti rotor (Beckman Coulter, CA, USA) for 70 minutes at $4{ }^{\circ} \mathrm{C}$. The pellets were washed with 11 $\mathrm{ml}$ of PBS by ultracentrifugation at $210,000 \times \mathrm{g}(35,000 \mathrm{rpm})$ using the SW41Ti rotor for 70 minutes at $4{ }^{\circ} \mathrm{C}$ and resuspended in around $250 \mu \mathrm{l}$ of PBS. To determine the size distribution of EVs, nanoparticle tracking analysis was carried out using the NanoSight system (Malvern Instruments, Malvern, UK) on samples diluted 100-fold with PBS. The system focuses a laser beam through a suspension of the particles of interest. Particles are visualized by light scattering using a conventional optical microscope perpendicularly aligned to the beam axis, which collects the light scattered from every particle in the field of view. A 60 -second video records all events for further analysis by the nanoparticle tracking analysis software (NTA2.3). The Brownian motion of each particle is tracked between frames to calculate its size using the Stokes-Einstein equation. Camera gain and temperature were set at value 13 and approximately $25{ }^{\circ} \mathrm{C}$, respectively.

\section{Immunoblot analysis}

The EV protein concentration was determined by Qubit Protein Assay Kit (Thermo Fisher Scientific, MA, USA). Equal amounts of EV protein were loaded onto 4-15\% Mini-PROTEAN TGXTM gels (BioRad, CA, USA). Following electrophoresis (100 V, $30 \mathrm{~mA})$, the proteins were transferred to a polyvinylidene difluoride membrane. The membranes were blocked with Blocking One solution (Nacalai Tesque, Kyoto, Japan) and then incubated with primary antibodies. For the detection of EV markers ${ }^{[11]}$, antibodies specific for CD63 (clone 8A12; Cosmo Bio, Tokyo, Japan), CD81 (clone 12C4; Cosmo Bio), Annexin A2 (clone 5/Annexin II; BD Biosciences, NJ, USA), and HSP70 (clone 7/Hsp70; BD Biosciences) were used as primary antibodies. After washing, the membrane was incubated with horseradish peroxidase-conjugated sheep anti-mouse IgG (Cytiva, Tokyo, Japan) and then subjected to enhanced chemiluminescence using ImmunoStar LD (Wako, Osaka, Japan). CD63 and CD81 were detected under non-reducing conditions.

\section{$R N A$ extraction and next-generation sequencing}

Total RNA was extracted from EVs using miRNeasy Mini Kit (Qiagen, Hilden, Germany). The extracted RNA was subjected to sequencing library construction using NEB Next Ultra II Directional RNA Library Prep Kit for Illumina (New England Biolabs, MA, USA) with NEB Next rRNA Depletion Kit (Human/Mouse/Rat) according to the manufacturer's protocols. The quality of the libraries was assessed using Agilent 2200 TapeStation High Sensitivity D1000 (Agilent Technologies, CA, USA). The pooled libraries of the samples were sequenced using NextSeq 500 (Illumina, CA, USA) in 76-base-pair single-end reads. Sequencing adaptors, low quality reads, and uncalled bases were trimmed with Trimmomatic- 0.38 tool ${ }^{[12]}$. The sequence reads were aligned to the Human reference genome (hg38) using STAR 2.7.1 $\mathrm{a}^{[13]}$. The aligned reads were subjected to downstream analyses using StrandNGS 3.4 software (Agilent Technologies, CA, USA). The read counts allocated for each gene and transcript (Ensembl Genes 2016.12.01) were quantified using a Trimmed Mean of M-value (TMM) method ${ }^{[14]}$.

\section{Gene expression data analysis}

Gene expression levels were evaluated based on the normalized read counts obtained by the next-generation sequencing. Gene expression signatures were assessed using gene set enrichment analysis (GSEA) and over-representation analysis (ORA) of WebGestalt (http://www.webgestalt.org/) by focusing on the biological process ontology in the Gene Ontology knowledgebase (http://geneontology.org/).
Statistical analysis

Statistical analyses were conducted using R software, developed by $\mathrm{R}$ Development Core Team at $\mathrm{R}$ Foundation for Statistical Computing (https://www.r-project.org/).

\section{Results}

\section{Characterizing EVs prepared from stem cell conditioned media}

EVs were prepared from the conditioned culture media of dental pulp-derived human MSCs (DP-hMSCs) and adipose tissue-derived human MSCs (A-hMSCs), and characterized regarding their particle counts and size distributions (Figure 1A). The particle counts for DPhMSCs-derived EVs (DP-EVs) and A-hMSCs-derived EVs (A-EVs) were determined to be $1.30 \times 10^{11}$ particles $/ \mathrm{ml}$ and $1.01 \times 10^{11}$ particles $/ \mathrm{ml}$, respectively. The particle sizes were peaked at $83 \mathrm{~nm}$ for DP-EVs and $102 \mathrm{~nm}$ for A-EVs. Immunoblotting analysis revealed the presence of conventional EV marker proteins CD63, CD81, ANXA2, and HSP70 in both DP-EVs and A-EVs, whereas HSP70 was less in A-EVs (Figure 1B). The data indicate the successful isolation of EVs from the conditioned culture media of hMSCs by ultracentrifugation method. Furthermore, the reproducibility of the method was evidenced by the constant detection of high levels of CD63 protein using ELISA and ExoScreen in multiple batches of EV preparations (data not shown).
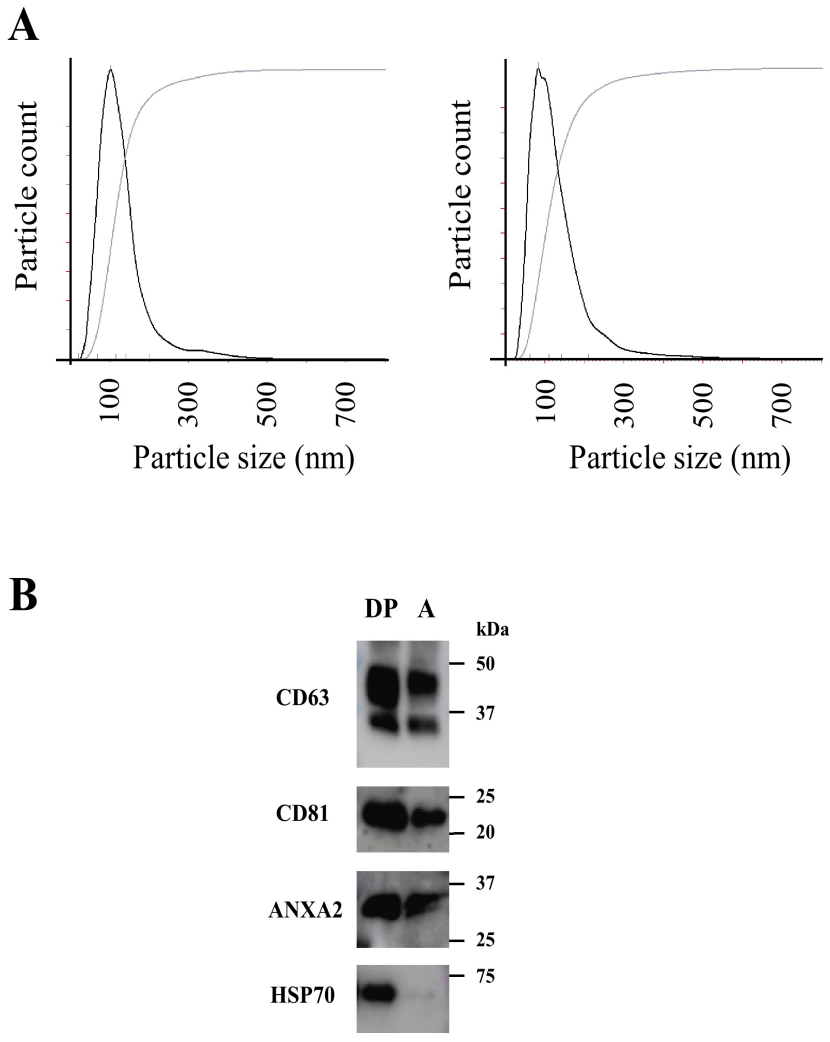

Figure 1: Characterization of EVs derived from DP-hMSCs and A-hMSCs (A) Particles in conditioned culture media of DP-hMSCs (left) and A-hMSCs (right) were analyzed using NanoSight. Black lines indicate particle size distributions. Gray lines indicate accumulated counts.

(B) EVs derived from DP-hMSCs (DP) and A-hMSCs (A) were analyzed via immunoblot analysis using antibodies against conventional EV markers; CD63, CD81, ANXA2 and HSP70. A 200 ng sample of EV proteins was used for the detection of CD63 and CD81. ANXA2 and HSP70 were detected using 500 ng of EV proteins. 
Transcripts in MSC-EVs of different tissue types

RNAs were isolated from DP-EVs and A-EVs and subjected to nextgeneration sequencing. Transcripts for 12,112 genes were identified commonly in both of DP-EVs and A-EVs while those for 1,220 and 1,387 genes were identified exclusively in DP-EVs and A-EVs, respectively (Figure 2, left). In total, transcripts for 14,719 genes were identified either in DP-EVs, A-EVs, or both. For analyzing gene expression signatures, 1,195 genes with the lowest expression values (normalized read counts for DP-EVs and A-EVs combined were two or less) were removed and the remaining 13,524 genes were subjected to GSEA (Figure 2, right). Genes expressed exclusively in DP-EVs (759 genes) or A-EVs (707 genes) were subjected to ORA.
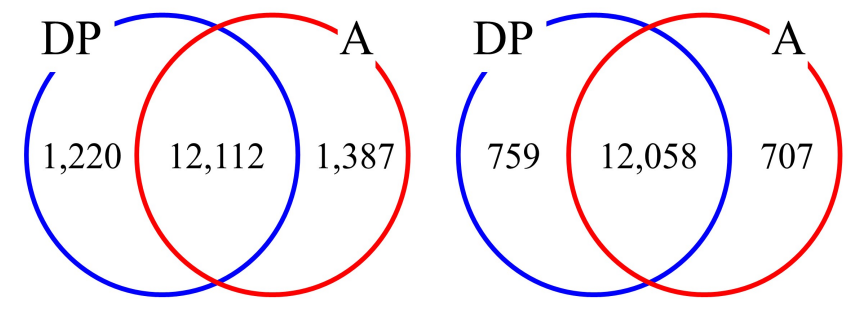

Figure 2: Numbers of expressed genes in EVS Numbers of genes, of which transcripts were identified in EVs of DP-hMSCs (DP) or A-hMSCs (A), are shown. The diagram on the left shows the numbers of all genes identified by next-generation sequencing. The diagram on the right shows the numbers of genes that were identified with the normalized read counts of three or more.

GSEA demonstrated that DP-EVs are characterized by the signatures of neural activity, negative chemotaxis, and cardiovascular development (Figure 3A, blue bars). Signatures of translation and mitochondrial activity were associated with A-EVs (Figure 3A, orange bars). ORA demonstrated signatures of neurogenesis in DPEVs, consistent with the GSEA results, with particular emphasis on retina and eye morphogenesis (Figure 3B). Signatures of skeletal system development were evident in A-EVs (Figure 3C).

\section{Comparing transcriptomes of MSC-EVs and hMSCs}

Since MSC-EVs are generated and released from hMSCs, the transcriptome of MSC-EVs may resemble that of the hMSCs. To determine whether this is the case, transcriptomes of MSC-EVs and hMSCs were compared. Previously, we identified 3,760 genes differentially expressed between DP-hMSCs and A-hMSCs [10]. Among the 3,760 genes, 3,010 were identified also in the current analysis on MSC-EVs. Using those 3,010 genes, relative expression values between DP-EVs and A-EVs were compared with those between DP-hMSCs and A-hMSCs. As shown in Figure 4A, the relative expression values for MSC-EVs positively correlated with those for hMSCs, indicating the transcriptomic similarity between MSC-EVs and hMSCs.

We further examined whether signature genes that characterize DPhMSCs and A-hMSCs are preferentially expressed in DP-EVs and AEVs, respectively. In the previous study, DP-hMSCs showed distinct gene expression signatures of neurogenesis with 18 signature genes that encode secreted factors. A-hMSCs, on the other hand, demonstrated gene expression signatures of angiogenesis with 26 signature genes that encode secreted factors ${ }^{[10]}$.
A

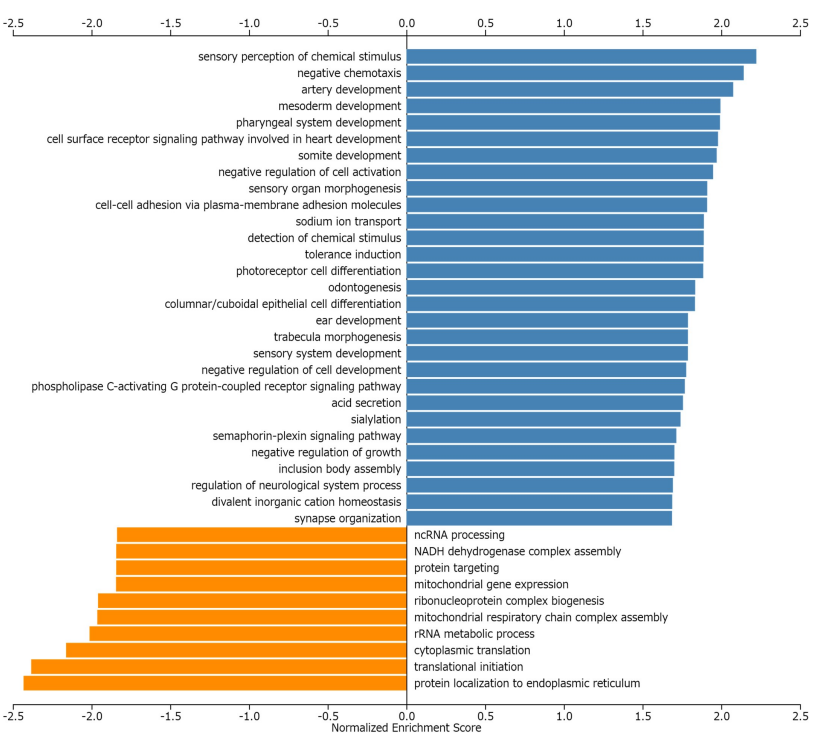

B

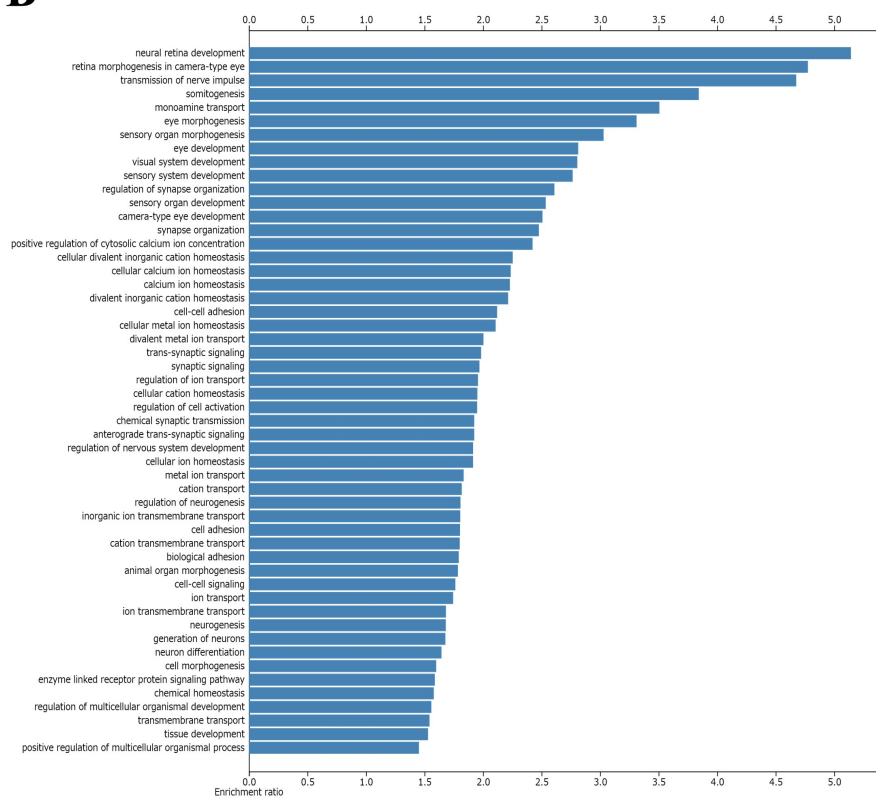

C

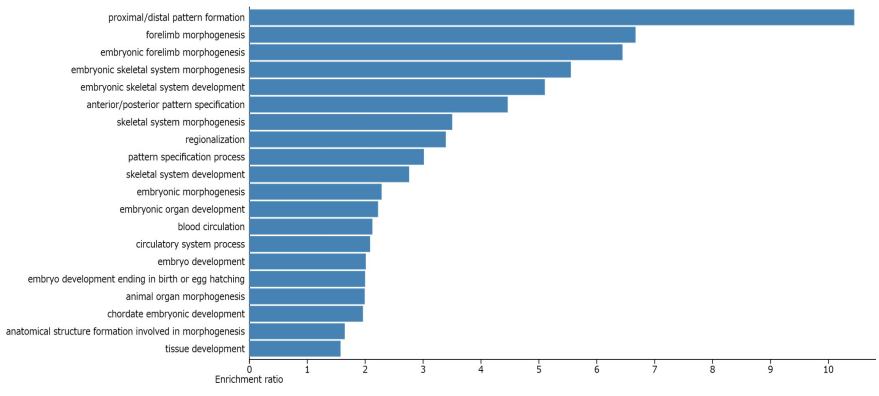

Figure 3: Gene ontology terms characterizing DP-EVS and A-EVS Transcriptomic signatures in DP-EVs and A-EVs were identified by gene set analyses. (A) GSEA identified gene ontology terms associated with DP-EVS and A-EVs. Values represent normalized enrichment scores significantly higher in DP-EVs (blue bars) or A-EVs (orange bars); FDR $\leq 0.05$. (B, $C$ ) ORA identified gene ontology terms significantly enriched in DP-EVS $(B)$ and $A-E V S(C)$. Values represent enrichment ratios; FDR $\leq 0.05$. 
A

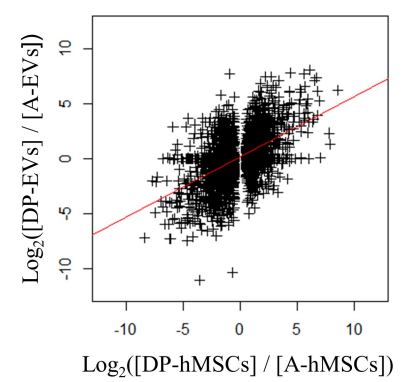

B

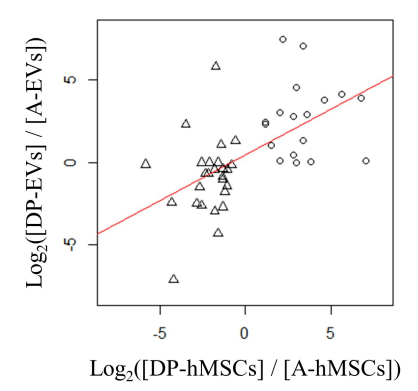

Hair growth factors

\begin{tabular}{|c|c|c|}
\hline Gene ID & DP & A \\
\hline HGF & 5.8 & 7.6 \\
\hline VEGFA & 8.6 & 7.5 \\
\hline FGF7 & 6.9 & 7.1 \\
\hline FGF2 & 6.6 & 6.8 \\
\hline IGF1 & 0.0 & 4.9 \\
\hline FST & 3.9 & 4.7 \\
\hline PDGFA & 2.9 & 3.7 \\
\hline LEP & 0.0 & 2.4 \\
\hline
\end{tabular}

Dermal matrices

\begin{tabular}{|c|c|c|}
\hline Gene ID & DP & A \\
\hline COL1A1 & 14.3 & 15.8 \\
\hline COL3A1 & 14.2 & 15.2 \\
\hline ELN & 0.0 & 6.1 \\
\hline
\end{tabular}

Figure 4: Gene expression profiles in $h M S C S$ and $M S C-E V S$ (A) The relative expression values between DP-EVS and $A-E V S, \log _{2}$ ([DP-EVS] / $\left.\left[A-E V_{S}\right]\right)$, were plotted against the relative expression values between DPhMSCs and A-hMSCs, $\log _{2}([D P-h M S C s] /[A-h M S C s])$, for 3,010 genes that were differentially expressed between DP-hMSCs and A-hMSCs. The values for MSC-EVs and hMSCs showed a positive correlation (coefficient $=0.52, P$ $\left.<2.2 \times 10^{-16}\right)$. (B) The relative expression values were plotted for $18 \mathrm{DP}$ hMSCs signature genes (open circle) and 26 A-hMSCs signature genes (open triangle) shown in Figure 5. The values for MSC-EVS and hMSCs displayed a positive correlation (coefficient $=0.60, P=1.1 \times 10^{-5}$ ).

In the current study on MSC-EVs, most of the 18 DP-hMSCs signature genes of neurogenesis were higher in DP-EVs than in AEVs (Figure 5, blue), and many of the 26 A-hMSCs signature genes of angiogenesis were higher in A-EVs than in DP-EVs (Figure 5, yellow). The relative expression values of these signature genes positively correlated between MSC-EVs and hMSCs to further illustrate their transcriptomic similarity (Figure 4B). Additionally, genes associated with hair growth and dermal matrices, known to be expressed in A-hMSCs, were highly expressed in A-EVs as well (Figure 6).

Neurogenesis
\begin{tabular}{|c|c|c|}
\hline Gene ID & hMSCs & EVs \\
\hline NRG1 & 2.17 & 7.48 \\
\hline BDNF & 3.39 & 7.10 \\
\hline FLRT3 & 2.98 & 4.54 \\
\hline SNCA & 5.61 & 4.12 \\
\hline CBLN2 & 6.77 & 3.94 \\
\hline PTN & 4.60 & 3.82 \\
\hline WNT5A & 2.03 & 3.05 \\
\hline NDP & 3.60 & 2.90 \\
\hline NETO1 & 2.79 & 2.82 \\
\hline SEMA3A & 1.17 & 2.45 \\
\hline DSCAM & 1.19 & 2.32 \\
\hline BMP7 & 3.40 & 1.32 \\
\hline SCN1B & 1.50 & 1.06 \\
\hline SPON2 & 2.81 & 0.44 \\
\hline NCAM1 & 7.04 & 0.10 \\
\hline SLIT3 & 2.00 & 0.09 \\
\hline KIRREL3 & 3.80 & 0.07 \\
\hline NTF3 & 3.00 & 0.00 \\
\hline
\end{tabular}

\section{Angiogenesis}

\begin{tabular}{|c|c|c|}
\hline Gene ID & hMSCs & EVs \\
\hline SFRP2 & -4.20 & -7.11 \\
\hline EREG & -1.58 & -4.30 \\
\hline FAP & -1.78 & -2.97 \\
\hline ADM2 & -1.29 & -2.69 \\
\hline CCL26 & -2.55 & -2.60 \\
\hline AP0D & -2.85 & -2.47 \\
\hline CCL2 & -4.30 & -2.42 \\
\hline HGF & -1.20 & -1.76 \\
\hline NDNF & -2.68 & -1.50 \\
\hline SRPX2 & -1.06 & -1.40 \\
\hline ANG & -1.33 & -1.03 \\
\hline VASH2 & -1.34 & -0.85 \\
\hline TEK & -2.34 & -0.69 \\
\hline EMCN & -2.15 & -0.69 \\
\hline FN1 & -1.05 & -0.42 \\
\hline FGF18 & -1.79 & -0.41 \\
\hline C3 & -1.31 & -0.39 \\
\hline FGF2 & -0.79 & -0.13 \\
\hline CHI3L1 & -5.84 & -0.12 \\
\hline CMA1 & -2.56 & 0.00 \\
\hline HYAL1 & -2.11 & 0.00 \\
\hline IL1B & -1.60 & 0.00 \\
\hline BMP2 & -1.40 & 1.07 \\
\hline FLT4 & -0.56 & 1.32 \\
\hline BMP6 & -3.48 & 2.32 \\
\hline PGF & -1.73 & 5.80 \\
\hline
\end{tabular}

Figure 5: Signature genes characterizing DP-hMSCs and A-hMSCs

Numbers in the ' $h M S C s$ ' columns represent $\log _{2}$ ratios for the $m R N A$ levels in DP-hMSCs vs. A-hMSCs Positive values (blue shade) indicate higher levels in DP-hMSCs than in AhMSCs. Negative values (yellow shade) indicate higher levels in A-hMSCs than in DP-hMSCs. Numbers in the ' $E V S$ ' columns represent $\log _{2}$ ratios for the $m R N A$ levels in DP-EVs vs. A-EVs.

Positive values (blue shade) indicate higher levels in $D P$-EVs than in A-EVS. Negative values (yellow shade) indicate higher levels in $A-D V$ s than in DPhMSCs.
Figure 6: Transcripts in EVS for hair growth factors and dermal matrices Numbers represent normalized read counts (in log scale), which indicate expression levels of genes encoding hair growth factors (left) and dermal matrices (right) in DP-EVs (DP) and A-EVS $(A)$.

\section{Discussion}

Recent studies have started to reveal the tissue-specific characteristics for $\mathrm{MSC}-\mathrm{EVS}^{[15,16]}$. Our current study sought to investigate the MSC-EVs of dental pulp and adipose tissue, and demonstrated that they possess distinct transcriptomic characteristics indicative of their potential therapeutic targets. Most evidently, DP-EVs revealed distinct transcriptomic signatures of neurogenesis and neural functions just like DP-hMSCs. This may suggest that DPEVs and DP-hMSCs are suitable for treating disorders that require regeneration or improved functions of neural system such as Alzheimer's and Parkinson's diseases. These diseases are associated with dysfunction in brain-derived neurotrophic factor (BDNF) signaling pathway. Our studies have demonstrated that BDNF is highly expressed in DP-hMSCs ${ }^{[10]}$ and DP-EVs (Figure 5, left). It is tempting to speculate that BDNF, abundant in DP-EVs, can ameliorate the neurodegenerative disorders ${ }^{[17]}$. Besides, strong signatures of neural retina development in DP-EVs may imply its potential in treating retinal diseases. Most of the angiogenesis signature genes for A-hMSCs were also expressed highly in A-EVs (Figure 5, right). Among them, SFRP have been suggested for its potential in treating myocardial infarction ${ }^{[18]}$ and muscle atrophy ${ }^{[19]}$.

The transcriptomic similarity between MSC-EVs and MSCs of the same tissue type was evident in the current study. This finding suggests the potential utility of MSC-EVs in replacing some of the therapeutic applications of MSCs. MSC-EVs can be an attractive alternative due to its low risk for embolism or rejection. We have been using the EVs-rich hMSCs conditioned media, StemSup ${ }^{\circledR}$ (Biotherapy Institute of Japan, Tokyo, Japan), in clinical settings to observe promising outcomes. Many patients treated with conditioned media of DP-hMSCs, which are rich with DP-EVs, have been experiencing improved eyesight, better sleep quality, and amelioration of numbness or pain on the limbs. A-hMSCs conditioned media, which are rich with A-EVs, have been used successfully for treating androgenetic alopecia and skin aging.

MSC-EVs have promising potential for treating various disorders. Novel therapeutic strategies for hard-to-treat disorders can be designed by understanding unique properties of MSC-EVs originated from various tissue types so that MSC-EVs with the most suitable characteristics are chosen for targeting the disease. 


\section{References}

1. Wang J, Chen Z, Sun M, Xu H, Gao Y, Liu J, Li M. Characterization and therapeutic applications of mesenchymal stem cells for regenerative medicine. Tissue Cell. 2020;64:101330.

2. Sagaradze GD, Basalova NA, Efimenko AY, Tkachuk VA. Mesenchymal stromal cells as critical contributors to tissue regeneration. Front Cell Dev Biol. 2020;8:576176.

3. Maqsood M, Kang M, Wu X, Chen J, Teng L, Qiu L. Adult mesenchymal stem cells and their exosomes: Sources, characteristics, and application in regenerative medicine. Life Sci. 2020;256:118002.

4. Tsiapalis D, O'Driscoll L. Mesenchymal stem cell derived extracellular vesicles for tissue engineering and regenerative medicine applications. Cells. 2020;9(4):991.

5. Brown C, McKee C, Bakshi S, Walker K, Hakman E, Halassy S, Svinarich D, Dodds R, Govind CK, Chaudhry GR. Mesenchymal stem cells: Cell therapy and regeneration potential. J Tissue Eng Regen Med. 2019;13(9):1738-1755.

6. Li CY, Wu XY, Tong JB, Yang XX, Zhao JL, Zheng QF, Zhao GB, Ma ZJ. Comparative analysis of human mesenchymal stem cells from bone marrow and adipose tissue under xeno-free conditions for cell therapy. Stem Cell Res Ther. 2015;6(1):55.

7. Xu L, Liu Y, Sun Y, Wang B, Xiong Y, Lin W, Wei Q, Wang H, He W, Wang B, Li G. Tissue source determines the differentiation potentials of mesenchymal stem cells: a comparative study of human mesenchymal stem cells from bone marrow and adipose tissue. Stem Cell Res Ther. 2017;8(1):275.

8. Rohban R, Pieber TR. Mesenchymal stem and progenitor cells in regeneration: Tissue specificity and regenerative potential. Stem Cells Int. 2017;2017:5173732.

9. Berebichez-Fridman R, Montero-Olvera PR. Sources and clinical applications of mesenchymal stem cells: State-of-theart review. Sultan Qaboos Univ Med J. 2018;18(3):e264e277.

10. Terunuma A, Ashiba K, Takane T, Sakaguchi Y, Terunuma H. Comparative transcriptomic analysis of human mesenchymal stem cells derived from dental pulp and adipose tissues. J Stem Cells Regen Med. 2019;15(1):8-11.
11. Clotilde Théry, Kenneth W Witwer, Elena Aikawa, et al Minimal information for studies of extracellular vesicles 2018 (MISEV2018): a position statement of the International Society for Extracellular Vesicles and update of the MISEV2014 guidelines. J Extracell Vesicles. 2018; 7: 1535750

12. Bolger AM, Lohse M, Usadel B. Trimmomatic: a flexible trimmer for Illumina sequence data. Bioinformatics, 2014;30(15): 2114-2120.

13. Dobin A, Davis CA, Schlesinger F, Drenkow J, Zalenski C, Jha S, Batut P, Chaisson M, Gingeras TR. STAR: ultrafast universal RNA-seq aligner. Bioinformatics. 2013;29(1):1521.

14. Robinson MD, Oshlack A. A scaling normalization method for differential expression analysis of RNA-seq data. Genome Biol. 2010;11(3):R25.

15. Wang ZG, He ZY, Liang S, Yang Q, Cheng P, Chen AM. Comprehensive proteomic analysis of exosomes derived from human bone marrow, adipose tissue, and umbilical cord mesenchymal stem cells. Stem Cell Res Ther. 2020;11(1):511.

16. Pelizzo G, Avanzini MA, Icaro Cornaglia A, Silvestri AD, Mantelli M, Travaglino P, Croce S, Romano P, Avolio L, Iacob G, Dominici M, Calcaterra V. Extracellular vesicles derived from mesenchymal cells: perspective treatment for cutaneous wound healing in pediatrics. Regen Med. 2018;13(4):385-394.

17. Mohammadi A, Amooeian VG, Rashidi E. Dysfunction in brain-derived neurotrophic factor signaling pathway and susceptibility to schizophrenia, Parkinson's and Alzheimer's diseases. Curr Gene Ther. 2018;18(1):45-63.

18. He W, Zhang L, Ni A, Zhang Z, Mirotsou M, Mao L, Pratt RE, Dzauet VJ. Exogenously administered secreted frizzled related protein 2 (Sfrp2) reduces fibrosis and improves cardiac function in a rat model of myocardial infarction. Proc Natl Acad Sci U S A. 2010;107(49):2111021115.

19. Zhu X, Kny M, Schmidt F, Hahn A, Wollersheim T, Kleber C, Weber-Carstens S, Fielitz J. Secreted frizzledrelated protein 2 and Inflammation-Induced skeletal muscle atrophy. Crit Care Med. 2017;45(2):e169-e183.

\section{List of Abbreviations}

$\begin{array}{ll}\text { MSCs: } & \text { Mesenchymal Stem Cells } \\ \text { DP-hMSCs: } & \text { Dental Pulp-Derived human Mesenchymal Stem Cells } \\ \text { A-hMSCs: } & \text { Adipose Tissue-Derived human Mesenchymal Stem Cells } \\ \text { EVs: } & \text { Extracellular Vesicles } \\ \text { MSC-EVs: } & \text { Mesenchymal Stem Cell-Derived Extracellular Vesicles } \\ \text { DP-EVs: } & \text { Dental Pulp-Derived human Mesenchymal Stem Cells-Derived Extracellular Vesicles } \\ \text { A-EVs: } & \text { Adipose Tissue-Derived human Mesenchymal Stem Cells-Derived Extracellular Vesicles } \\ \text { GSEA: } & \text { Gene Set Enrichment Analysis } \\ \text { ORA: } & \text { Over-Representation Analysis }\end{array}$

Funding

This work was supported by Asfreya Co., Ltd. (Osaka, Japan).

\section{Corresponding Author}

Atsushi Terunuma, Tsukuba Memorial Hospital, 1187-299 Kaname, Tsukuba, Ibaraki 300-2622, Japan

Email: terunuma_a@tsukuba-kinen.or.jp 